\title{
A new approach to tolerance analysis based on tracking local coordinate systems
}

\author{
Xiaochen ZHAI*, Qungui DU*, Wenxi WANG**, Qi WEN*, Baoshan LIU* and Dongwen ZHU* \\ *School of Mechanical\& Automotive Engineering, South China University of Technology \\ Wushan RD, Tianhe District, Guangzhou 510641, China \\ E-mail: zhaixiaochen@126.com \\ ${ }^{*}$ School of International Studies, Sun Yat-sen University \\ Sun Yat-sen University Zhuhai Campus, Tangjiaw an, Zhuhai 519000, China
}

Received: 6 October 2016; Revised: 27 March 2017; Accepted: 8 May 2017

\begin{abstract}
Among current approaches to tolerance analysis in three-dimensional space, the Matrix method based on rigid body kinematics is widely used. However, this method ignores the impact a tolerance feature (TF) may get from the variations of TFs before it, therefore showing its demerits when applied to long and complex dimension chains. For this reason, this paper puts forward a new tolerance analysis model where two local coordinate systems are built at each TF: a nominal one based on the datum of current TF and a tracking one based on the variations of the prior TFs. Then the variations of all TFs along the dimension chain are accumulated. Compared with the Matrix method, the new approach generates results with more accuracy and higher efficiency.
\end{abstract}

Key words :Matrix method, Tolerance analysis, Tracking local coordinate system, Nominal local coordinate system, Tolerance accumulation

\section{Introduction}

In product manufacturing, mechanical components are very likely to have machining errors which may adversely impact the product performance. The refore, in the design phase, designers usually introduce tolerance to limit the erro rs with in a certain range. They also conduct tolerance analysis to determine whether the tolerance meets users' requirements or not.

In three-dimensional space, the component features that contain errors are also called Tolerance Features (TFs) (Whitney, 2004). TFs may vary from their nominal positions, such variations including small translations and rotations. Hence, tolerance analysis should also be conducted in three-dimensional space. At present, the most widely-adopted analysis methods include the Matrix method put forward by (Desrochers and Rivière, 1997; Marziale and Polini, 2009), and the Jacobian Torsor method presented by (Desrocher et al., 2003; Ghie, 2009). Both of them are based on the rigid body kinematics which translates the variations of TFs to that of local coord inate systems of the TFs, and ultimately obtains the error transmission and accumulation. These two methods both require to establish local coordinate system at the nominal position of each TF, but they also have differences. The Matrix method adopts the Homogeneous Transform Matrix (HTM) to describe the s mall variations of TFs, through which it gets the contribution of all TF variations to the target feature, and then accumulates the contributions to the total coordinate system. In contrast, the Jacobian Torsor method adopts the open-loop kinematic chains to map each TF to the target feature, thereby obtaining the variation of the target feature.

Through comparis on, it can be found that both the Matrix method and the Jacobian-Torsor method are centered on the HTM. The latter is in its nature another expression form of the former. Since the Matrix method is applicable and easy to conduct, it has been widely used in a number of CAT software (Prisco and Giorleo, 2002; Salomons et al., 1995). However, this method ignores the geometrical and physical meanings of error transmission. In fact, when machining errors are considered, the local coordinate systems tied to TFs are unlikely to be at the ideal positions, and their positional and orientational correlation with the total coordinate system is not nominal, either. Therefore, the Matrix method does not apply to the actual situation of tolerance transmission, and the result it generates may greatly 
deviate from the actual one. For these reasons, it is necessary to perform tolerance analysis in local coordinate systems which take errors into consideration.

The second section of this paper proposes a way to establish tracking local coordinate systems mentioned above. Following this, the third section presents a tolerance analysis model based on the tracking coordinate systems. The fourth section verifies this model through an example, and the fifth section draws a conclusion.

\section{Establishment of Tracking Coordinate Systems}

In order to describe the establishment of tracking coordinate systems in a more direct way, Figure 1 gives an example of a simple assembly composed of Block A and Block B. Specifically, Fig ure 1 (a) shows a coordinate system of the as sembly without considering errors, while Fig. 1 (b) indicates the situation when errors are considered. N stands for the top surface of Block A; P represents the top surface of Block B. Position TA and parallelis $\mathrm{m}$ TB are tolerance specifications for $\mathrm{N}$, and position TC and paralle lis $\mathrm{m}$ TD are tolerance specifications for $\mathrm{P}$. $O_{1}$ is the nominal local coordinate systemon $\mathrm{N}$; $\mathrm{O}_{2}$ is the nominal local coordinate systemon P.

In Fig. 1 (a), both $O_{1}$ and $O_{2}$ have fixed positions and orientations independent from each other. Their positional and orientational correlations with the total coordinate system $O$ can be expressed as $P_{O_{1} \rightarrow O}$ and $P_{O_{2} \rightarrow O}$. However, in actual situations, TA and TB have impacts on both $O_{1}$ and $O_{2}$. As shown in Fig. 1 (b), the black solid line stands for the nominal position and orientation; the blue solid line shows the actual position and orientation. When N moves, Block B deviates from the nominal position and orientation to the actual ones, and $\mathrm{O}_{2}$ also varies to $\mathrm{O}_{2}$ ' accordingly. That way, $P_{\mathrm{O}_{2} \rightarrow O}$ becomes meaningless. It should be noted that the situation shown in Fig. 1 is only one of the numerous variations.

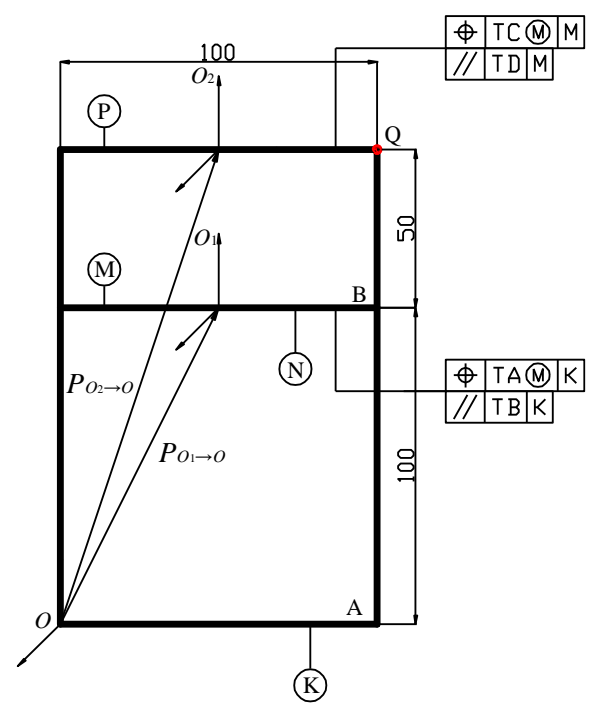

(a) Coordinate system without considering errors

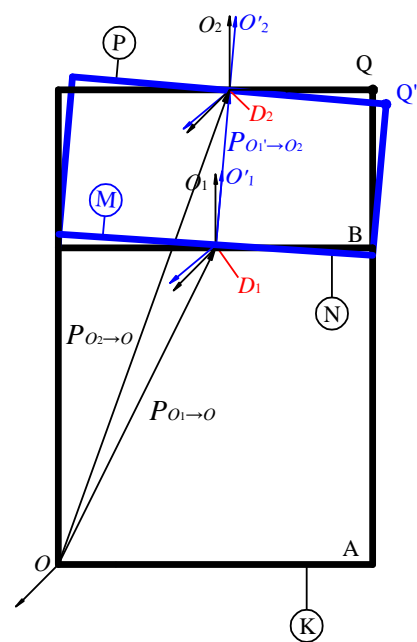

(b) Coordinate system considering errors

Fig. 1 Simple as sembly composed of BlockA and Block B

As discussed in Section 1, the traditional Matrix method establishes only nominal coordinate systems for tolerance analysis, therefore failing to reveal the variation relations between the assembly parts and their variation ranges. In a more practical way, errors caused by the variations from $\mathrm{TF}_{1}$ to $\mathrm{TF}_{(i-1)}$ should be considered when the coordinate system $O_{i}$ is established at $\mathrm{TF}_{i}$, and the positional and orientational correlation between $O_{i}$ and $O$ can therefore be obtained. Hence, this paper proposes to establish two local coordinate systems at each TF: a nominal coordinate system and a tracking coordinate system. The former $\left(O_{i}\right)$ is used to describe the variation of $\mathrm{TF}_{i}$, and the latter $\left(O_{i}{ }^{\prime}\right)$ is used to transmit the variation of $\mathrm{TF}_{i}$ to the subsequent $\mathrm{TF}_{i+1}$, and takes the variation as the datum of $\mathrm{TF}_{i+1}$. In this way, the error caused by $\mathrm{TF}_{i}$ can be taken into consideration when the coordinate system $O_{i+1}$ of $\mathrm{TF}_{i+1}$ is established. When $i$ increases from 1 to the whole length of the dimension chain $(n)$, the impacts of all TFs on the target feature can be acquired.

The specific process of establishing tracking coordinate systems is described as below:

(1) Determine the TFs that have impacts on the target feature. Based on the assembly and datum relationships 
between these TFs, establish a dimension chain where a total coordinate system $O$ sits at the beginning, and the target feature for tolerance analysis resides at the end.

(2) Establish a nominal coordinate system $O_{i}$ and a tracking coordinate system $O_{i}$ 'at $\mathrm{TF}_{i}$ in sequence.

(3) Determine the positional and orientational correlation between $O_{i}$ ' and $O . O_{i}$ ' can track and record the errors generated from $\mathrm{TF}_{1}$ to $\mathrm{TF}_{(i-1)}$ along the dimension chain, and its positional and orientational correlation with $O$ is expressed by $P_{O_{i}^{\prime} \rightarrow O}$. Similarly, the positional and orientational correlation between $O_{i}$ and $O$ is indicated by $P_{O_{i} \rightarrow O}$.

Take the variation transform $D_{i}$ into consideration, then $P_{O_{i}^{\prime} \rightarrow O}$ can be determined as follows:

$$
P_{O_{i}^{\prime} \rightarrow O}=P_{O_{i} \rightarrow O} \cdot D_{i}
$$

The variation of each TF describes the relative positional uncertainty of TFs with respect to a set of data. According to the shape generation processes proposed in the paper (Sugimura et al., 2012), the HTM method in kinematics of mechanis $m$ can be used to represent the variations of TFs. The variations of each TF contain 6 independent parameters. Each of these parameters defines a displacement in itself which will be referred to as elementary displacement. The rotation displacements will be expressed as angles of rotation around the nominal local coordinate system axis; angles $\alpha, \beta, \gamma$ around the $\mathrm{x}, \mathrm{y}, \mathrm{z}$ axis; The translation displacements will be expressed respectively by components $\mathrm{u}, \mathrm{v}, \mathrm{w}$, which translate along the $\mathrm{x}, \mathrm{y}$, and $\mathrm{z}$ axis.

The corresponding homogeneous transform matrix describing the six elementary displacements can be written as follows:

$$
\begin{aligned}
T\left(u_{i}\right) & =\left[\begin{array}{llll}
1 & 0 & 0 & u_{i} \\
0 & 1 & 0 & 0 \\
0 & 0 & 1 & 0 \\
0 & 0 & 0 & 1
\end{array}\right], T\left(v_{i}\right)=\left[\begin{array}{llll}
1 & 0 & 0 & 0 \\
0 & 1 & 0 & v_{i} \\
0 & 0 & 1 & 0 \\
0 & 0 & 0 & 1
\end{array}\right], T\left(w_{i}\right)=\left[\begin{array}{cccc}
1 & 0 & 0 & 0 \\
0 & 1 & 0 & 0 \\
0 & 0 & 1 & w_{i} \\
0 & 0 & 0 & 1
\end{array}\right], \\
\operatorname{Rot}\left(\alpha_{i}\right) & =\left[\begin{array}{cccc}
1 & 0 & 0 & 0 \\
0 & \cos \alpha_{i} & -\sin \alpha_{i} & 0 \\
0 & \sin \alpha_{i} & \cos \alpha_{i} & 0 \\
0 & 0 & 0 & 1
\end{array}\right] \operatorname{Rot}\left(\beta_{i}\right)=\left[\begin{array}{cccc}
\cos \beta_{i} & 0 & \sin \beta_{i} & 0 \\
0 & 1 & 0 & 0 \\
-\sin \beta_{i} & 0 & \cos \beta_{i} & 0 \\
0 & 0 & 0 & 1
\end{array}\right] \operatorname{Rot}\left(\gamma_{i}\right)=\left[\begin{array}{cccc}
\cos \gamma_{i} & -\sin \gamma_{i} & 0 & 0 \\
\sin \gamma_{i} & \cos \gamma_{i} & 0 & 0 \\
0 & 0 & 1 & 0 \\
0 & 0 & 0 & 1
\end{array}\right]
\end{aligned}
$$

Every variation transform $D_{i}$ can be described as the product of six elementary displacements $T\left(u_{i}\right), T\left(v_{i}\right), T\left(w_{i}\right), \operatorname{Rot}\left(\alpha_{i}\right), \operatorname{Rot}\left(\beta_{i}\right), \operatorname{Rot}\left(\gamma_{i}\right)$. The Roll-Pitch and Yaw convention has been adopted for the order of these components, the multiplication order is as follows:

$$
D_{i}=T\left(u_{i}\right) \times T\left(v_{i}\right) \times T\left(w_{i}\right) \times \operatorname{Rot}\left(\alpha_{i}\right) \times \operatorname{Rot}\left(\beta_{i}\right) \times \operatorname{Rot}\left(\gamma_{i}\right)
$$

In general, according to the types of TFs and datum, suitable components should be selected from the six elementary displacements for multiplication. For example, in terms of a plane feature with position tolerance requirements as shown in Fig. 2(a), its variation transform $D_{i_{-} P}$ has three degrees of freedom (DOFs) (Takahashi et al., 2014), including a translational DOF along the $Z_{i}$-axis and two rotational DOFs along the $X_{i}$-axis and $Y_{i}$-axis, respectively. After variation $D_{i_{-} P}$, the vertexes A, B, C, D of the plane vary to A', B', C', and D', and the nominal coordinate system $O_{i_{-}} P$ moves to the tracking coordinate system $O^{\prime}{ }_{i} P$. Furthermore, Figure 2(b) shows a cylinder feature with position tolerance requirements (Sugimura et al., 2012). Its variation transform $D_{i_{-} C}$ has four DOFs, including two translational DOFs along the $X_{i}$ - axis and $Z_{i}$-axis, and two rotational ones along the $X_{i}$-axis and $Z_{i}$-axis, respectively. After variation $D_{i_{-} C}$, the vertexes $\mathrm{E}$ and $\mathrm{F}$ become E' and F', and the nominal coordinate system $O_{i_{-} C}$ also varies to the position of the tracking coordinate system $O^{\prime}{ }_{i} C$. Some other feature types and expression forms of $D_{i}$ are listed in the references (Ghie, 2009), the corresponding ways of establishing tracking coordinate systems are similar to what mentioned above. 


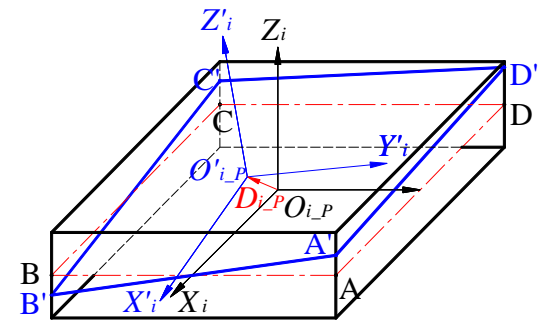

(a) Plane feature

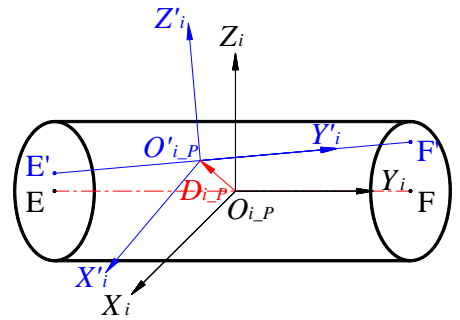

(b) Cylinder feature

Fig. 2 Feature variations with errors

Apart from plane feature and cylinder feature, there are also five other types of TFs a mong the most common ones. Table 1 shows these five types and their corresponding variation transform matrix $D$.

Table1 Description of the other five types of TFs

\begin{tabular}{|c|c|c|}
\hline Type of TFs & Figure & Variation transform matrix $D$ \\
\hline $\begin{array}{c}\text { General } \\
\text { surface } \\
\text { (3-translation } \\
\text { 3-rotaion) }\end{array}$ & 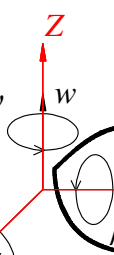 & $T(u) \times T(v) \times T(w) \times \operatorname{Rot}(\alpha) \times \operatorname{Rot}(\beta) \times \operatorname{Rot}(\gamma)$ \\
\hline $\begin{array}{l}\text { Prismatic } \\
\text { surfaces } \\
\text { (2-translation } \\
\text { 3-rotaion) }\end{array}$ & & $T(u) \times T(w) \times \operatorname{Rot}(\alpha) \times \operatorname{Rot}(\beta) \times \operatorname{Rot}(\gamma)$ \\
\hline $\begin{array}{l}\text { Surfaces of } \\
\text { revolution } \\
\text { (3-translation } \\
\text { 2-rotaion) }\end{array}$ & & $T(u) \times T(v) \times T(w) \times \operatorname{Rot}(\alpha) \times \operatorname{Rot}(\gamma)$ \\
\hline $\begin{array}{c}\text { Helical } \\
\text { surfaces } \\
\text { (3-translation } \\
\text { 2-rotaion) }\end{array}$ & & $T(u) \times T(v) \times T(w) \times \operatorname{Rot}(\alpha) \times \operatorname{Rot}(\gamma)$ \\
\hline $\begin{array}{l}\text { Spherical } \\
\text { surfaces } \\
\text { (3-translation } \\
\text { 0-rotaion) }\end{array}$ & & $T(u) \times T(v) \times T(w)$ \\
\hline
\end{tabular}

(4) Determine the positional and orientational correlation between the nominal coordinate system $O_{i+1}$ and the 
total coordinate system $O$.

After the variation transform $D_{i}, \mathrm{TF}_{i}$ deviates to the actual position and orientation, and $O_{i}$ that is tied to $\mathrm{TF}_{i}$ varies to $O_{i}{ }^{\prime}$. When mechanical parts are assembled, $O_{i}{ }^{\prime}$ of $\mathrm{TF}_{i}$ becomes the datum reference system of $\mathrm{TF}_{i+1}$ because of the datum relationships between parts. Then based on the nominal geometric relationship between $\mathrm{TF}_{i}$ and $\mathrm{TF}_{i+1}$, the positional and orientational correlation between $O_{i+1}$ and $O_{i}{ }^{\prime}$ can be determined as follows:

$$
P_{O_{i+1} \rightarrow O}=P_{O_{i}^{\prime} \rightarrow O} \cdot P_{O_{i+1} \rightarrow O_{i}^{\prime}}
$$

where: $P_{O_{i+1} \rightarrow O}$ stands for the positional and orientational correlation between $O_{i+1}$ of $\mathrm{TF}_{i+1}$ and $O . P_{O_{i}^{\prime} \rightarrow O}$ indicates that between $O_{i}{ }^{\prime}$ of $\mathrm{TF}_{i}$ and $O$, and $P_{O_{i+1} \rightarrow O_{i}^{\prime}}$ is that between $O_{i+1}$ of $\mathrm{TF}_{i+1}$ and $O_{i}$ '.

\section{Tole rance Analysis Model Based on Tracking Coordinate Sys tems}

In Section 2, we have established nominal coordinate systems and tracking coordinate systems along the dimension chain from $\mathrm{TF}_{1}$ to $\mathrm{TF}_{n}$, realizing accumulation of errors. When the target feature $\mathrm{TF}_{n}$ does not have any tolerance requirements, the positional and orientational correlation expression $P_{O_{n} \rightarrow O}$ is

$$
P_{O_{n} \rightarrow O}=\prod_{i=1}^{n-1} P_{O_{i} \rightarrow O} \cdot D_{i} \cdot P_{O_{i+1} \rightarrow O_{i}^{\prime}}
$$

Accordingly, in the total coordinate system $O$, the variation expression $\Delta M$ of target feature $M$ is

$$
\Delta M=P_{O_{n} \rightarrow O} \cdot[M]_{O_{n}}
$$

When the target feature $\mathrm{TF}_{n}$ has tolerance requirements, the positional and orientational correlation expression $P_{O_{n} \rightarrow O}$ is

$$
P_{O_{n}^{\prime} \rightarrow O}=\left(\prod_{i=1}^{n-1} P_{O_{i} \rightarrow O} \cdot D_{i} \cdot P_{O_{i+1} \rightarrow O_{i}^{\prime}}\right) \cdot D_{n}
$$

The variation expression $\Delta M$ of target feature $\mathrm{M}$ becomes

$$
\Delta M=P_{O_{n}^{\prime} \rightarrow O} \cdot[M]_{O_{n}}
$$

where: $[M]_{O_{n}}$ is the coordinate value of $\mathrm{M}$ in $O_{n}$.

In actual situations, among all the TFs along the dimension chain, only the first one has nominal position and orientation in the total coordinate system $O$. Therefore, the method of linear accumulation is not applicable for tolerance analysis. Instead, multiplication should be adopted, which explains why this paper conducts tolerance analysis based on tracking local coordinate systems. What's more, the new approach avoids the problem of repeated error accumulation met by the Matrix method. According to tolerance semantics, when a TF is constrained by several tolerances instead of motivated by them separately, we only need to ensure that the TF is within each tolerance zone.

\section{Application of the Method}

The Fig. 3(a) shows a three-part centering pin mechanis $m$ consisting of Base, Pin and Block whose specifications are given in Fig. 4(Ghie, 2009). In this mechanism, Q is the vertex of the Pin and also the target feature; K is the surface of the Base. The vertical distance between $\mathrm{Q}$ and $\mathrm{K}$ is indicated by $\Delta Q \quad(-0.5 \mathrm{~mm},+0.5 \mathrm{~mm})$. In order to meet the functional requirement, researchers designate the tolerance specification in Table 2 below. 


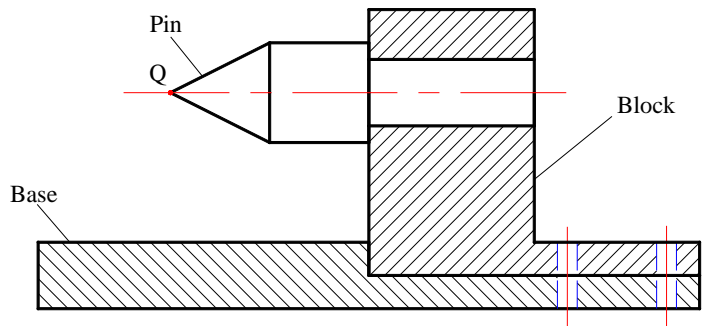

(a) Nominal as sembly

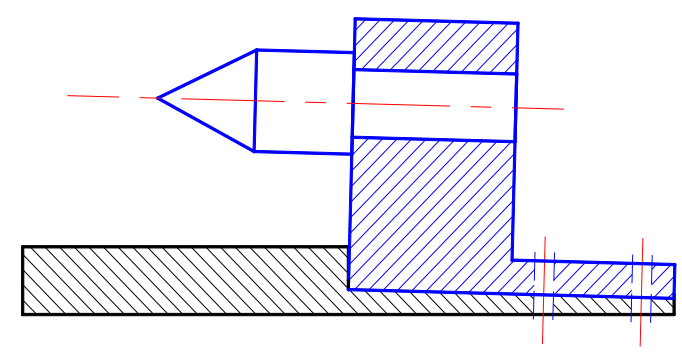

(b) As sembly considering errors

Fig. 3 A three-part centering pin mechanism

Table 2 Tolerance specification designated by researchers (Unit: $\mathrm{mm}$ )

\begin{tabular}{c|c|c|c|c|c|c}
\hline \hline $\mathrm{ta}$ & $\mathrm{tb}$ & $\mathrm{tc}$ & $\mathrm{td}$ & $\mathrm{te}$ & $\mathrm{tf}$ & $\mathrm{tg}$ \\
\hline 0.2 & 0.1 & 0.2 & 0.1 & $\mathrm{H} 11: 0.00 / 0.13$ & $\mathrm{H} 8:-0.033 / 0.000$ & 0.1 \\
\hline
\end{tabular}
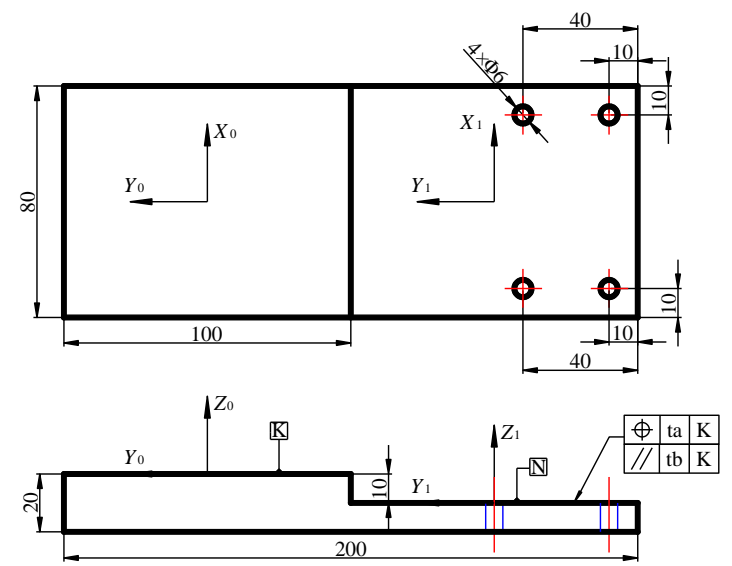

(a) Base
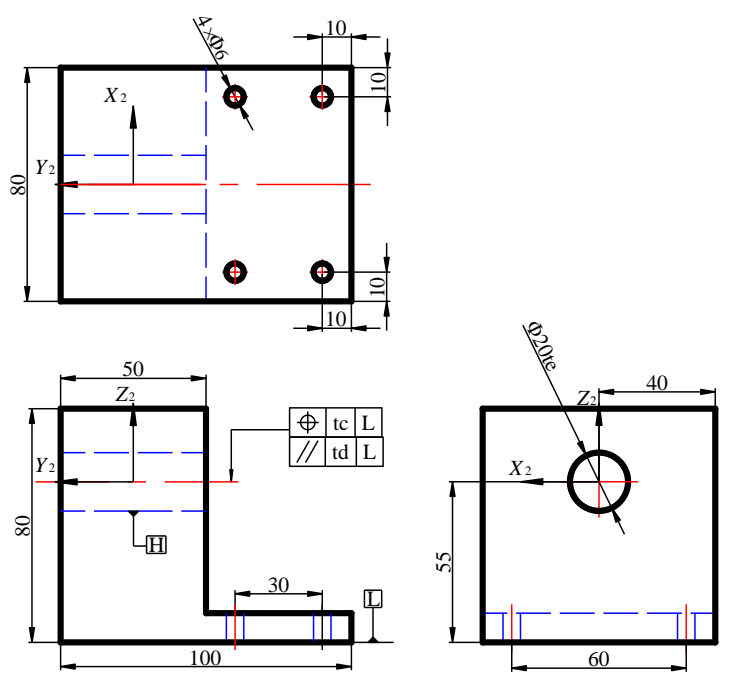

(c) Block

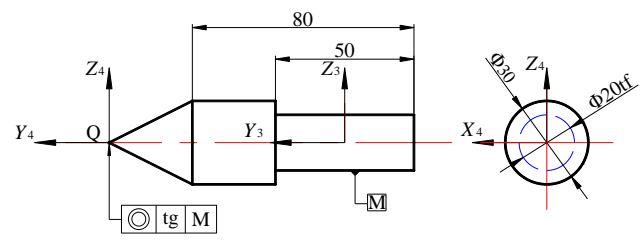

(b) Pin

Fig. 4 Specifications of the three parts

In the following part, we apply the tracking coordinate system to tolerance analysis and verify its validity.

First, connect the TFs that have contributions to $\Delta Q$ based on the dimension relations between components and the datum relations between tolerances. A dimension chain is therefore established. As shown in Fig. 5, the solid arrow indicates the assembly sequence. N, H, M and Q are TFs of the centering pin; $\mathrm{K}$ is the common datum of position tolerance ta and paralle lis $m$ tolerance tb on surface $N$ of the Base. Surface $N$ of the Base and surface L of the Block have no tolerance and totally fit with each other. Position tolerance tc and parallelis $\mathrm{m}$ tolerance td of hole $\mathrm{H}$ are both based on L. The mating of surface $\mathrm{M}$ and hole $\mathrm{H}$ is constrained by fit tolerances te and tf. Point $\mathrm{Q}$ on the Pin is constrained by the coaxiality tolerance tg relative to $\mathrm{M}$. When errors are taken into consideration, the situation of the centering pin is shown as in Fig. 3(b). It should be noted that it is only one of the many assembly situations with errors.

Following that, build a total coordinate system $R_{0}$ on the datum $\mathrm{K}$. Establish nominal coordinate systems $R_{1}, R_{2}$, $\mathrm{R}_{3}, \mathrm{R}_{4}$ and tracking coordinate systems $\mathrm{R}_{1}{ }_{1}, \mathrm{R}_{2}{ }_{2}, \mathrm{R}_{3}{ }_{3}, \mathrm{R}_{4}{ }_{4}$ at features $\mathrm{N}, \mathrm{H}, \mathrm{M}$, and Q, respectively. The connection order of the coordinate systems is indicated by the red dotted arrow in Fig. 5. Then determine the positional and orientational correlations of each nominal or tracking coordinate system with the total coordinate system. Since there is a tolerance requirement for the target feature Q, Equation (6) in Section 3 is adopted, through which we can get the expression of 
$\Delta Q$ as follows:

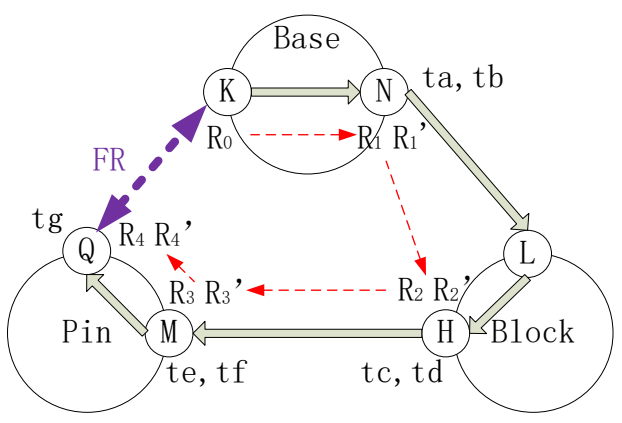

Fig. 5 Dimension chain of the centering pin

$$
\Delta Q=\prod_{i=1}^{3}\left(P_{R_{1} \rightarrow R} \cdot D_{i} \cdot P_{R_{i+1} \rightarrow R_{i}^{\prime}}\right) \cdot D_{4} \cdot[Q]_{R_{4}}
$$

Finally, use inequalities to express the constraints provided by the tolerance zone. This way, the solution to the result of $\Delta Q$ can be translated to an optimal problem under constraints. There are altogether 15 variation transform factors during the optimization, namely, $\alpha_{1}, \beta_{1}, w_{1}, \alpha_{2}, \gamma_{2}, u_{2}, w_{2}, \alpha_{3}, \gamma_{3}, u_{3}, w_{3}, \alpha_{4}, \gamma_{4}, u_{4}, w_{4}$. In this example, since $\mathrm{N}, \mathrm{H}$ and $\mathrm{M}$ all contain multiple tolerance requirements, the variations of each one of them have complex coupling relationship, which makes the initial values of the variations unavailable and the calculation complicated. Therefore, this paper adopts the Genetic Algorithm for non-linear programming in MATLAB software. This method combines genetic algorith $\mathrm{m}$ with non-linear programming algorith $\mathrm{m}$, so that the merits of these two algorithms can be exploited to the full. To be specific, the genetic algorithm is used for global search and nonlinear programming algorithm for local search. This way, we can obtain the optimal results. Below is the algorithm flow chart:

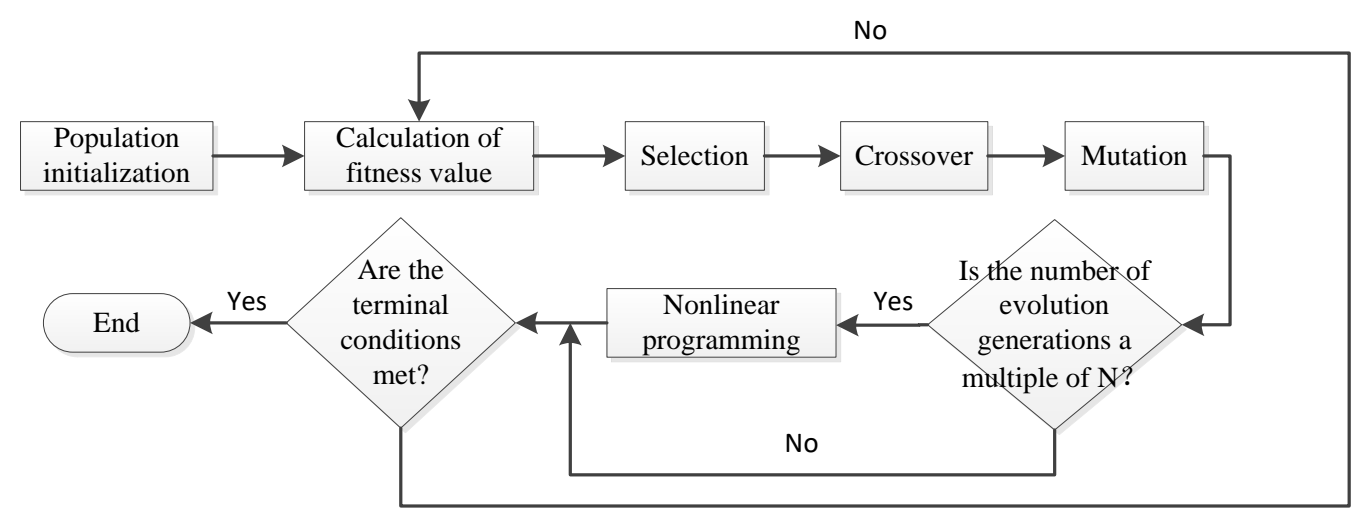

Fig. 6 Algorithm flow chart

With the optimal solution, the variation range of $\Delta Q$ is $\pm 0.8289 \mathrm{~mm}$ using the method of tracking local coordinate systems, while the result is $\pm 1.1361 \mathrm{~mm}$ using the Matrix method. The paper (Ghie, 2009) adopted Jacobian Torsor method, and it obtained a result of $\pm 0.976 \mathrm{~mm}$. Hence, it can be seen that none of these three methods can make the tolerance design meet the requirement. We conclude that the tolerances values (Table 2) may be expanded to ensure the manufacturing costs to be cheaper.

It can be seen that the range of $\Delta Q$ is expanded using the latter two methods, The discrepancy lies in that the Matrix method establishes local coordinate systems in a different way from the new approach proposed by this paper, which affects the accuracy of error accumulation. What's more, the Matrix method repeatedly calculates variations 
generated by different tolerance requirements for a TF, therefore expanding the range of $\Delta Q$. By comparison with the traditional Matrix method, the new tolerance analysis model based on tracking coordinate systems is proven to be effective and applicable.

\section{Conclusion}

This paper probes into tolerance analysis models which are based on rigid body kinemics. It analyzes the establishment of local coord inate systems, and puts forward a new approach to tolerance analysis based on tracking coordinate systems. The new model creatively establishes two local coord inate systems at each TF: one at the nominal position based on the datum of a TF, the other at the actual position of the TF when errors are considered. In this way, errors can be tracked by the local coordinate systems. The new approach shows the following advantages:

(1) It takes full consideration of the possible variations of TFs in the tolerance zone, and takes into account variation impacts of a TF on the later TFs.

(2) Compared with the Matrix method, the new approach adopts multiplication operation and avoids the problem of repeated tolerance accumulation. When there are multiple tolerance constraints for a TF, the mapping from each tolerance to the total coordinate system is calculated only once. The calculation is therefore simplified and accuracy improved.

(3) It can consider a local coordinate system at any TF as a total coordinate system, and figure out the re lationship between this coordinate systemand otherTFs without establishing new dimension chains.

However, this paper does not discuss the contribution of form tolerance which, when considered, requires to build more complex coordinate systems and error transmission systems. It will be explored in the future work.

\section{References}

Desrocher, A., Ghie, W. and Laperriere, L., Application of a Unified Jacobian - Torsor Model for Tolerance Analysis, Journal of Computing and Information Science in Engineering, Vol.3, No.1 (2003), pp.2-14.

Desrochers, A. and Rivière, A., A matrix approach to the representation of tolerance zones and clearances, International Journal of Advanced Manufacturing Technology, Vol.13, No.9 (1997), pp.630-636.

Ghie, W., Statistical analysis tolerance using jacobian torsor model based on uncertainty propagation method, The International Journal of Multiphysics, Vol.3, No.1 (2009), pp.11-30.

Marziale, M. and Polini, M., A review of two models for tolerance analysis of an assembly: vector loop and matrix, International Journal of Advanced Manufacturing Technology, Vol.43, No.11-12 (2009), pp.1106-1123.

Prisco, U. and Giorleo, G., Overview of current CAT systems, Integrated Computer-Aided Engineering, Vo1.9, No.4 (2002), pp.373-387.

Salomons, O. W., Poerink, H. J. Jonge., Slooten, F.van., Houten, F. J. A. M. van. and Kals, H. J. J., A Computer Aided Tolerancing Tool based on Kinematic Analogies, Springer, (1995), pp.47-70.

Sugimura, N., Watabiki, H., Thasana, W., Iwamura, K. and Tanimizu, Y., Analysis of Kinematic Motion Deviations of Rotary Tables Based on Geometric Tolerances, Journal of Advanced Mechanical Design, Systems, and Manufacturing, JSME, Vol. 6, No. 7(2012), pp.1132-1142.

Takahashi, A., Yoshida, A., Thas ana, W., Sugimura, N., Iwamura, K. and Tanimizu, Y., Analysis of Kinematic Motion Deviations of Machining Centers Based on Geometric Tolerances, Journal of Advanced Mechanical Design, Systems, and Manufacturing, JSME, Vol. 8, No. 4(2014), pp.1-12.

Whitney, D. E., Mechanical Assemblies - Their Design, Manufacture, and Role in Product Development (2004), Oxford University Press. 